\title{
Study of generation and trigger system of the Extracorporeal Cardiac Shock Wave
}

\author{
Liang Yin ${ }^{1,2}$ a, Ye Zhang ${ }^{1}$, Shouli Bai ${ }^{1,2}$, Xin Qi ${ }^{1,2}$ b* $^{*}$, Ying Zhou ${ }^{3}$ \\ ${ }^{1}$ College of Science, Beijing University of Chemical Technology, Beijing, 100029, China \\ ${ }^{2}$ Beijing Key Laboratory of Environmentally Harmful Chemical Analysis, Beijing University of \\ Chemical Technology, Beijing, 100029, China \\ ${ }^{3}$ Department of Cardiology, China-Japan Friendship Hospital, Beijing, 100029, China \\ ayinliang@mail.buct.edu.cn ${ }^{b^{*}}$ haitianyisebuct@sina.com \\ * please mark the corresponding author with an asterisk
}

\begin{abstract}
Keywords: coronary artery disease, extracorporeal cardiac shock wave, placing signage, trigger system, energy output formula.

Abstract. This article introduces the placing signage and the high-voltage trigger system of the extracorporeal cardiac shock wave treatment instrument. The placing signage is composed of a solenoid coil, a cylindrical sheet metal and a parabolic reflection cup. The cylindrical sheet metal is pushed sharply by electromagnetic force to generate a shock wave which can treat the myocardial ischemia symptoms with its mechanical force and cavitation in human tissues. The energy output formula of the placing signage is derived. The trigger system provides high-voltage electrical impulses to the electromagnetic coil to cause an electromagnetic induction of the placing signage. The trigger system includes high-voltage booster module, voltage control module, energy storage capacitor array, high voltage switch module, charging counting module and mixed signal processing module. The energy range of the shock wave is $2 \sim 7 \mathrm{~J}$ and the energy density of the focus is $0.004 \sim 0.413 \mathrm{~mJ} / \mathrm{mm}^{2}$. The extracorporeal cardiac shock wave system developed in this paper can emit stable period, narrow pulse width, low energy and high pressure shock wave.
\end{abstract}

\section{Introduction}

The Coronary Artery Disease (CAD) has done serious damage to people's life and health nowadays. Coronary Artery Bypass Graft (CABG) and Percutaneous Coronary Intervention (PCI) are two effective medical methods to treat $\mathrm{CAD}$ in addition to the drug treatment. However, they are not applicable to patients who have accepted surgical treatment or at end-stage. The Extracorporeal Cardiac Shock Wave Therapy (ECSWT) can promote angiogenesis, improve the symptoms of myocardial ischemia with its mechanical force and cavitation in human tissues and could be a new non-invasive treatment of CDA $[1,2]$.

The extracorporeal shock wave was applied for the treatment of urinary calculi in the 1980s. In vitro studies have demonstrated that ECSWT can enhance vascular endothelial growth factor (VEGF) mRNA expression in cultured human umbilical vein endothelial cells and in rat bone marrow cells (BMCs) as well as promote BMCs differentiation into cells with endothelial phenotype [3, 4]. Swiss company STORZ MEDICAL developed the first extracorporeal cardiac shock wave apparatus MODLITH SLC in the world in 2003 [5]. In 2010, Kikuchi performed a double-blind placebo-controlled trial in patients with severe angina pectoris to further confirm the effectiveness and safety of ECSWT [6]. Wang $\mathrm{Yu}$ et al. reported that ECSWT can reduce angina and improve myocardial function in patients with refractory coronary artery disease and ameliorate myocardial fibrosis by decreasing the amount of fibrocystic after acute myocardial infarction [7].

ECSWT is a non-invasive treatment [8]. So the emission energy of the shock wave should be strictly controlled to achieve the best therapeutic effect as well as reduce the damage to other tissues. The placing signage of the ECSWT is designed and the energy transfer and output formulas are derived, as well as the trigger system is studied in this paper. A high-voltage circuit is designed to charge a 
capacitor array and the electrical energy is converted into magnetic energy by LC oscillation system, then the cylindrical sheet metal is pushed sharply by the electromagnetic force to generate a shock wave. The experiment proved that the shock wave system can generate stable period, narrow pulse width, low energy and high pressure shock wave.

\section{Experiment}

\section{The principle of ECSWT}

The discussed model is given as follow: ECSWT can deliver a sequence of transient pressure disturbances characterized by high peak pressure $(100 \mathrm{MPa})$, fast pressure rise $(10 \mathrm{~ns})$, rapid propagation, and short lifecycle $(10 \mathrm{~ms})$ produced by an appropriate generator to the area of myocardial ischemia. Due to mechanical shear force and cavitation the ECSWT can increase the Vascular Endothelial Growth Factor (VEGF) and the Basal Fibroblast Growth Factor (BFGF), can promote neovascularization of cardiac tissue and significantly improve cardiac function in patients with severe CAD and refractory angina.

\section{The design of the placing signage}

The placing signage is studied in this paper. There are four kinds of placing signage according to the shock wave generation principle: electrohydraulic, piezoelectric, electromagnetic, micro detonation. The more commonly used are electrohydraulic and electromagnetic. The principle of electromagnetic shock wave generation is that the cylindrical sheet metal is pushed sharply to produce shock waves by a force which generated by the electromagnetic induction between a high frequency coil and the sheet metal. Then the shock waves are reflected by a reflection cup and the energy is focused on the therapeutic target. The structure of the shock wave placing signage is shown in Fig. 1.

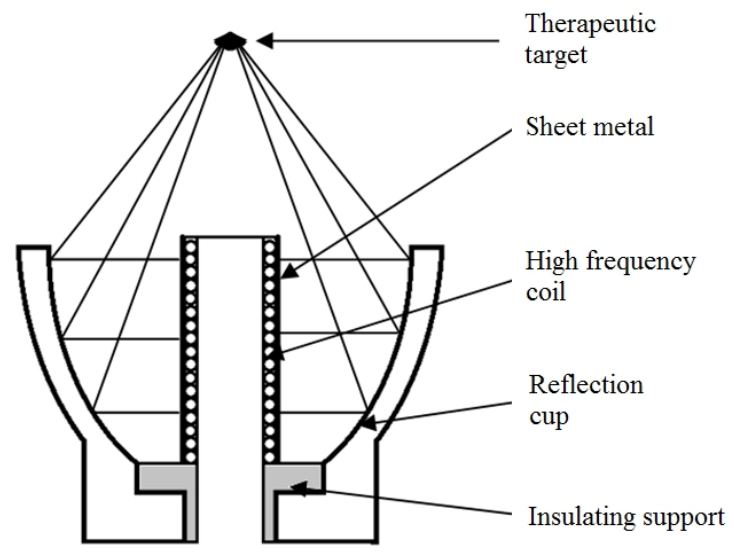

Fig. 1 The structure of the shock wave placing signage

A cylindrical hollow insulating support is installed in the center of the shock wave placing signage. Out of the insulating support a high frequency coil is winded around and a cylindrical sheet metal is installed out of the coil. Due to the electromagnetic induction principle, there will be a reverse induced current be produced in the sheet metal when a high pressure pulse cross the coil, resulting in a repulsive force to make the sheet metal rapidly expand to produce a shock wave. A parabolic reflection cup is installed out of the shock wave placing signage. The shock waves which spread through the media are mirrored and focused by the inner surface of the reflection cup on the target area. The treatment depth of the shock wave is about $15 \mathrm{~cm}$. The reflecting surface is got by rotating the parabola. The structure of reflection cup is shown in Fig. 2. 


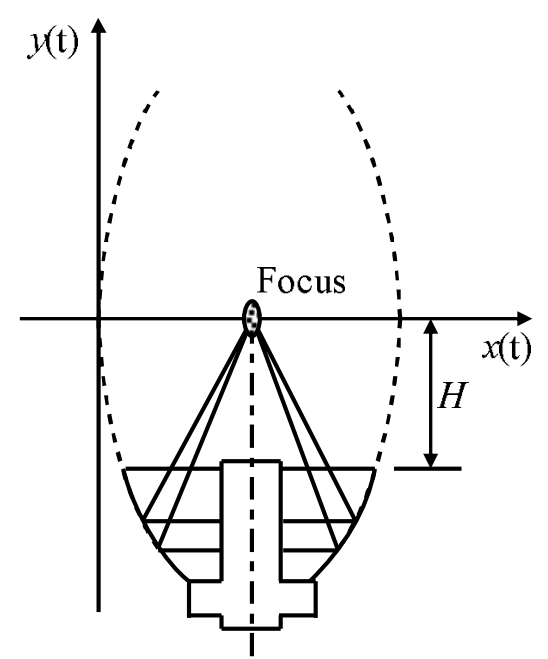

Fig. 2 The structure of reflection cup

\section{The calculation of the output energy of the placing signage}

The shock wave used to treat the CAD is a kind of sound waves. The attenuation and the shearing stress of the shock wave are small in human tissue and the penetrating power is strong. So the shock wave can reach treatment area stably. In the condition of no damping, the electric energy can be completely converted into magnetic energy in LC oscillation circuit. Compared with capacitance voltage, the phase advance of the capacitive current is $90^{\circ}$ and the phase delay of the inductance current is $90^{\circ}$. The phase difference between the electric energy and magnetic energy is $90^{\circ}$ in LC circuit. The variation tendency of $i_{\mathrm{C}}, u, i_{\mathrm{L}}$ in LC circuit is shown in Fig. 3.
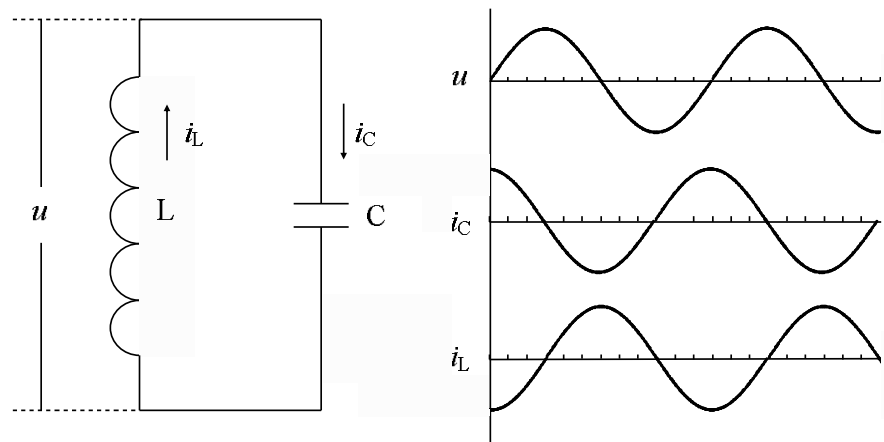

Fig. 3 Variation tendency of $i_{\mathrm{C}}, u, i_{\mathrm{L}}$ in LC circuit

When the high-voltage energy storage capacitor is charged with high-voltage power supply, the LC discharge circuit is closed and there will be an electromagnetic oscillation between the capacitance and inductor coil. The actual oscillation is damped, and the energy is consumed gradually, but the shock wave is completed in the first cycle of the LC oscillation. So it can be equal to simple harmonic oscillation, and the electrical energy is converted into magnetic energy. The frequency is:

$$
f=\frac{1}{2 \pi \sqrt{L C}}
$$

Where $C$ is the capacitance of the high-voltage energy storage capacitor; $L$ is the inductance of the inductor coil. $L$ can be got from Eq. 2:

$$
L=k \mu_{0} \mu_{s} N^{2} s / l
$$


Where $k$ is a coefficient; $\mu_{0}$ is the vacuum magnetic permeability; $\mu_{\mathrm{S}}$ is the relative permeability in the air; $N$ is the number of turns; $S$ is the cross area of the inductor coil; $l$ is the length of the inductor coil.

The total energy $E$ is lossless at simple harmonic oscillation circuit, and the electrical energy is equal to the magnetic energy:

$$
E=\frac{1}{2} C U_{m}^{2}=\frac{1}{2} L I_{m}^{2}
$$

So when the electrical energy is fully converted into magnetic energy, the current through the inductor $I_{\mathrm{m}}$ peak:

$$
I_{m}=\sqrt{C U^{2} / L}
$$

The stress amplitude of the shock wave near the sheet metal is:

$$
P_{M}=\mu \frac{N I_{\mathrm{m}}}{D}
$$

Where $D$ is the radius of the inductor coil; $I_{\mathrm{m}}$ is the pulse current; $N$ is the turns of the inductor coil. It can be seen that the pressure amplitude of the shock wave peaked when the current in the inductor coil reach the maximum.

The sheet metal drives the water medium to form sound waves. The sound intensity of the focal spot, namely, the average energy flow density is:

$$
I=\frac{\bar{W}}{S}=\frac{P_{e}^{2}}{\rho \cdot c}
$$

Where $P_{\mathrm{e}}$ is the root mean square of sound pressure; $\rho$ is the density of water; $c$ is the sound velocity in water.

It can be obtained from Eq. 1 to Eq. 6 that the energy of the focus can be controlled by controlling the charging voltage $U_{\mathrm{m}}$ when the capacitance and inductance are determinate.

So the values of high voltage and energy range can be calculated. The energy of the ECSWT is about 2 7 J, which is only one tenth of the energy for the treatment of lithotripsy.

The capacitance of the high-voltage capacitor is $0.7 \mu \mathrm{F}$, so it can be obtained from Eq. 3 that the high-voltage pulse value $U$ is $2.5 \sim 5.0 \mathrm{KV}$. The inductance of the air core coil is:

$$
\begin{aligned}
& L=k \mu_{0} \mu_{s} N^{2} s / l \\
& =0.67 \cdot 4 \pi \times 10^{-7} \cdot 1 \cdot 100^{2} \cdot 0.0028 / 0.06 \\
& =3.96 \times 10^{-4} \mathrm{H}
\end{aligned}
$$

It can be obtained that the wave crest of the shock wave pressure is $10 \sim 35 \mathrm{KPa}$, and the diameter of the focal spot is $3 \sim 8 \mathrm{~mm}$, and the energy density of the focus is $0.003 \sim 0.413 \mathrm{~mJ} / \mathrm{mm}^{2}$.

\section{Trigger system of the ECSWT}

Because of the periodic heart beats, the cycle of the shock wave should match the cycle of heart beat, namely the shock wave should work in the refractory period of heart break. The energy emitting frequency from the trigger system is $1 \mathrm{~Hz}$. After the capacitor array is charged, the capacitor discharges a microsecond voltage pulse. The electrical energy can be converted into magnetic energy to generate 
a shock wave when a high voltage pulse is applied on the high frequency coil. The high-voltage energy-storage capacitor is charged first, and then the electrical energy is discharged to the coil with a high voltage switch. The coil and capacitor compose a LC oscillation circuit.

Trigger system includes: high voltage booster module, the energy storage capacitor array, charging control module, counting module, high voltage switch module, mixed signal processing module etc. The system block diagram is shown in Fig. 4.

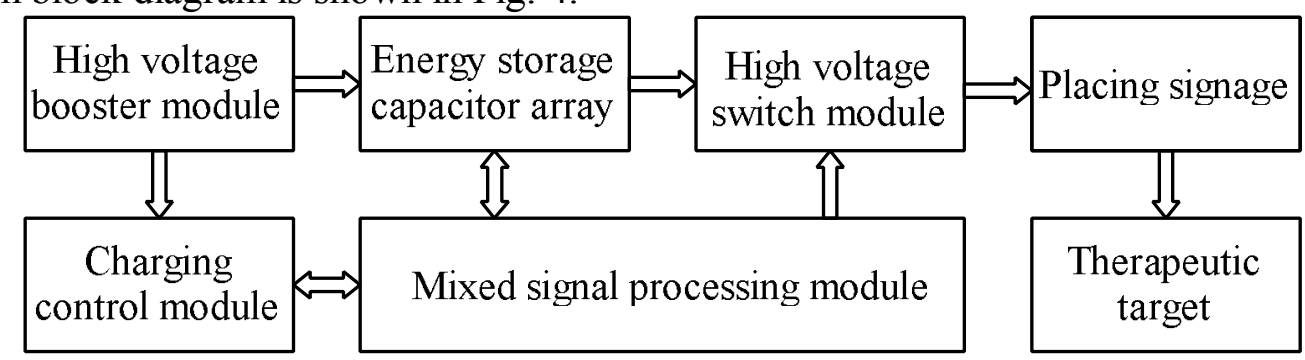

Fig.4: The block diagram of high voltage circuit system

\section{High voltage booster module}

The high voltage booster module is used to convert low DC voltage into high DC voltage based on the SG3525A chip and a transformer.

SG3525A is a PWM controller and reference voltage source output chip produced by ST Company, which can convert low DC voltage into AC voltage. A $12 \mathrm{~V} \mathrm{DC}$ voltage is used as the power supply of the low voltage side of SG3525A. The output of SG3525A is used to control the MOSFET and as the primary AC voltage input of the transformer. Then the transformer converts low AC voltage into high $\mathrm{AC}$ voltage and the high $\mathrm{AC}$ voltage is converted into high $\mathrm{DC}$ voltage with electrolytic capacitor.

\section{Energy storage capacitor array}

A capacitor array is used as the energy storage module. The capacitor array and the high frequency coil compose a LC oscillation circuit to convert the electrical energy into the magnetic energy. Some low value capacitors are connected in series and in parallel to compose a capacitor array to improve the withstand voltage and to control the capacitor value convenient. The capacitor array is shown in Fig. 5.

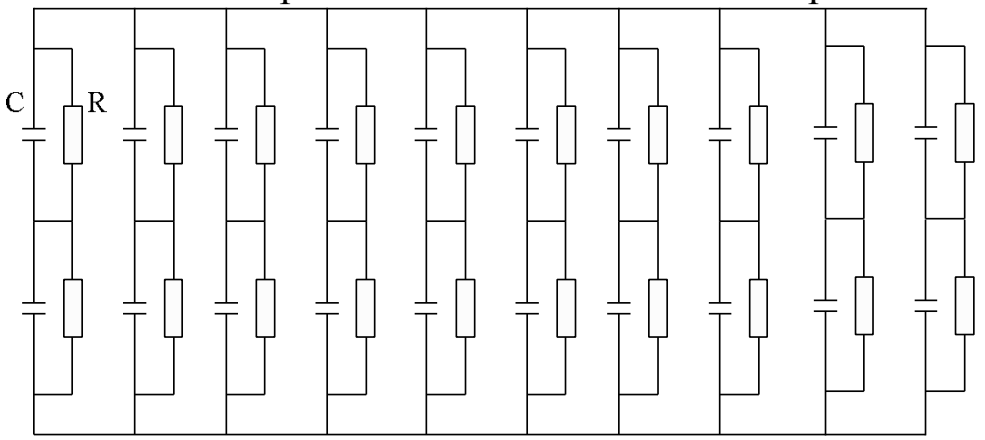

Fig. 5: Capacitor array

Twenty $200 \mathrm{nF}, 4 \mathrm{KV}$ voltage resistant, non-polar capacitors are linked together in parallel and serial, and each capacitor is parallel with a $1 \mathrm{M}$ resistance to release residual charge. High voltage and high temperature resistant nylon material are used as the base and fixing device of the capacitor array. A $1 \mu \mathrm{F}$ non-polar capacitor array is got and the voltage resistant can reach $8 \mathrm{KV}$ voltages.

The output voltage is $2575 \mathrm{~V}$ and the capacitance of the capacitor array is $1 \mu \mathrm{F}$, so the energy storage of the capacitor is:

$$
E=\frac{1}{2} C U^{2}=3.31 J
$$

It is in the energy range of the ECSWT of $2 \sim 7 \mathrm{~J}$. The rising edge of the shock waves produced is 0.7 $\mu \mathrm{s}$, and the half-width is $1.8 \mu \mathrm{s}$. 


\section{Conclusions}

The principle of the electromagnetic induction and the energy conversion of the LC oscillation circuit are used to design the placing signage of the ECSWT. The placing signage system can meet the design requirements according to the Theoretical Calculation.

The transformer and energy storage capacitor array are used to generate high voltage pulse, and a trigger system of the ECSWT is designed. The energy emitting frequency from the trigger system is 1 Hz. The system can convert electrical energy of the capacitor into magnetic energy of the electromagnetic coil rapidly to produce energy controllable shock wave with pulse width of $1.8 \mu$ s.

\section{References}

[1] C. Andrew, P. Megha, R.P. Martin, S.R. Guy, K. Darshak, N.D. Anthony, L. Amir, Safety and Efficacy of Extracorporeal Shock Wave Myocardial Revascularization Therapy for Refractory Angina Pectoris, Mayo Clinic Proceedings 89(2014) 346-354.

[2] R.G. Juan, L. Amir, Cardiac shock-wave therapy in the treatment of refractive angina pectoris, Journal of interventional cardiology 3(2011) 191-201.

[3] U. Toyokazu, I. Kenta, A. Kohtaro, Extracorporeal cardiac shock wave therapy improves left ventricular remodeling after acute myocardial infarction in pigs, Coronary Artery Disease 18(2007), 397-404.

[4] D.M. Franca, N. Daria, C. Clotilde, Cardiac Shock Wave Therapy: Assessment of Safety and New Insights into Mechanisms of Tissue Regeneration, Journal of Cellular and Molecular Medicine 16(2012) 936-994.

[5] Y. Wang, T. Guo, H.Y. Cai, Cardiac Shock Wave Therapy Reduces Angina and Improves Myocardial Function in Patients With Refractory Coronary Artery Disease, Journal of interventional cardiology 33(2010) 693-699.

[6] Y. Kikuchi, K. Ito, Y. Ito, Double-blind and Placebo-controlled Study of the Effectiveness and Safety of Extracorporeal Cardiac Shock Wave Therapy for Severe Angina Pectoris, Circulation journal 74(2010) 589-591.

[7] Y. Wang, T. Guo, T.K. Ma, A modified regimen of extracorporeal cardiac shock wave therapy for treatment of coronary artery disease, Cardiovascular Ultrasound 10(2012) 1-10.

[8] X.L. Zhang, X.Y. Yan, C.Y. Wang, The dose-effect relationship in extracorporeal shock wave therapy: the optimal parameter for extracorporeal shock wave therapy, Journal of surgical research $1(2014)$ 484-492. 